\title{
Finite difference approximation to the Cauchy problem for non-linear parabolic differential equations
}

\author{
by P. Besala (Gdańsk)
}

Franciszek Leja in memoriam

\begin{abstract}
An explicil finite difference scheme is used to approximate the solution of the initial value problem for non-linear second order parabolic equations in several independent variables. The approximated solution is allowed to belong to a "natural" class of fast increasing functions. An error estimate implying the convergence of the difference scheme is obtained.
\end{abstract}

1. Introduction. The problem of finite difference approximation to initial boundary value problems for parabolic equations in bounded domains has been investigated by many authors. In [3], [6], [9], [10] this problem is treated for non-linear equations in several independent variables, in bounded parallelepipeds. Numerical treatment of the Cauchy problem for parabolic equations is found in papers [1], [4], [5], [12], [13]. Among them only paper [1] is concerned with many independent variables. In [1], [4] the approximated solutions are assumed to be bounded. Further, in papers [5], [12], [13] the Cauchy problem for second order parabolic equations (just as in the earlier paper [8] ine Cauchy problem for first order hyperbolic equations) is treated with the "longitudinal" method of lines which reduces this problem to the corresponding problem for a countable system of ordinary differential equations. However, only in [12] the approximated solution may grow as fast as in our case. On the other hand, paper [12] deals only with the case of one idependent spatial variable and its nonuniform discretization.

Considering the line method approximation to be the limit case of the finite difference scheme as the difference quotient for the time variable tends to the derivative, we see that the line method cannot be applied under our circumstances because of the assumption about the relation between the sizes of the steps for the time and space variables $\left(k / h^{2} \geqslant d>0\right.$ in (8)).

We approximate the solution of the Cauchy problem for parabolic equations by the solution of a suitable discrete problem, using a uniform explicit difference scheme, and prove a theorem concerning the error estimate 
and the convergence of the scheme. We find, first of all, that the approximated solution is allowed to grow like $\exp \left(K|x|^{2}\right)$. This contains extensions of some the results of [1], [4] to the unbounded solutions case. Moreover, we consider non-linear equations, obtaining generalizations of the results of [3], [6], [9], [10] to the Cauchy problem.

Parabolic equations containing the mixed derivatives of the unknowns have been dealt with by A. Fitzke [3] and M. Malec [9], [10] under different assumptions on the function $f$ occurring in the given equation. M. Malec used seven-point approximations to the mixed derivatives which enabled him to make a relatively weak assumptions on $f$ - implying parabolicity.

In this paper we use the same approximations to the derivatives and make the same assumption of $f$ concerning parabolicity as those introduced by $\mathrm{M}$. Malec.

Let us finally mention that not long ago Z. Kowalski [7] proved the convergence of a difference scheme for non-linear elliptic equations under a more general, "almost usual" condition of ellipticity.

2. Let $x=\left(x_{0}, x_{1}, \ldots, x_{n}\right) \in R^{n+1}$ and $S=\left\{x \in R^{n+1}: 0 \leqslant x_{0} \leqslant T, T>0\right\}$. Consider the Cauchy problem

$$
u_{x_{0}}=f\left(x, u, u_{x}, u_{x x}\right) \quad \text { for } x \in S ; \quad u(x)=g(x) \text { for } x_{0}=0,
$$

where $u_{x}=\left(u_{x_{1}}, \ldots, u_{x_{n}}\right), u_{x x}=\left(u_{x_{i} x_{j}}\right)_{i, j=1}^{n}$.

We make the following assumptions:

I. Problem (1) has a solution $u(x)$ which is of class $C^{2}(S)$, has continuous third order derivatives with respect to variables $x_{i}(i=1, \ldots, n)$ in $S$ and satisfies the growth conditions

$$
|u|,\left|u_{x_{0} x_{0}}\right|,\left|u_{x_{i} x_{j} x_{s}}\right| \leqslant H(x ; M, K):=M \sum_{v=1}^{n} \exp \left(K x_{v}^{2}\right)
$$

for all $i, j, s=1, \ldots, n$ and for some constants $M, K>0$.

II. Function $f(x, u, q, r)$ is continuous for $(x, u, q, r) \in S \times R^{1+n+n^{2}}$ and of class $C^{1}$ in $u, q, r$. For any fixed pair of indices $i, j(i \neq j)$ the derivative $\partial f / \partial r_{i j}$ is always non-negative or always non-positive and $\partial f / \partial r_{i j}=\partial f / \partial r_{j i}$. Further, there exist constants $L_{0}, L_{1} \geqslant 0, L_{2} \geqslant \varkappa>0$ such that

$$
\begin{gathered}
|\partial f / \partial u| \leqslant L_{0}, \quad\left|\partial f / \partial q_{i}\right| \leqslant L_{1}, \quad\left|\partial f / \partial r_{i j}\right| \leqslant L_{2} \\
\partial f / \partial r_{i i}-\sum_{j=1, j \neq i}^{n}\left|\partial f / \partial r_{i j}\right| \geqslant \varkappa .
\end{gathered}
$$

Furthermore

$$
|f(x, 0,0,0)| \leqslant H(x ; M, K)
$$


11I. There exist constants $\lambda>0, N>K$ and $p \in(0,1)$ so that

$$
\lambda \geqslant 4 L_{2} \max \left\{n, N p^{-1} \exp \left[(N / 2) e^{\lambda T}+\lambda T\right]\right\} .
$$

Assumption III is a natural restriction on $K$ or $T$. It is well known that in general a solution of (1) belonging to the class of "fast increasing" functions exists only for small $x_{0}$ 's. We give two examples in which (6) is satisfied:

$1^{\circ}$ Arbitrary $T>0, \lambda \geqslant 4 n L_{2}$ and $p \in(0,1)$ while $K$ is so small that

$$
K p^{-1} \exp \left[(K / 2) e^{\lambda T}+\lambda T\right]<n .
$$

$2^{\circ}$ Arbitrary $K, N>K, p \in(0,1)$ and

$$
\lambda \geqslant 4 L_{2} \max \left\{n, N p^{-1} \exp (N e / 2+1)\right\} \quad \text { whereas } T \leqslant 1 / \lambda .
$$

Let $A$ be the set of vectors (multi-indices) $a=\left(\alpha_{0}, \alpha_{1}, \ldots, \alpha_{n}\right)$ such that $x_{0}=0,1, \ldots, l ; x_{i}=0, \pm 1, \pm 2, \ldots(1 \leqslant i \leqslant n), l$ being a positive integer. Let $A^{\prime}=A \cap\left(x_{0}<l\right)$. In $S$ we introduce the set $S^{\prime}$ of nodal points

where

$$
x^{a}=\left(x_{0}^{\alpha_{0}}, x_{1}^{\alpha_{1}}, \ldots, x_{n}^{\alpha_{n}}\right), \quad a \in A,
$$

$$
x_{0}^{\alpha_{0}}=x_{0} k \quad(k=T / l) \quad \text { and } \quad x_{i}^{\alpha_{i}}=x_{i} h \quad(h=\text { const }>0 ; i=1, \ldots, n) .
$$

Further we define

$$
i(a)=\left(\alpha_{0}, \ldots, \alpha_{i-1}, \alpha_{i}+1, \alpha_{i+1}, \ldots, \alpha_{n}\right), \quad 0 \leqslant i \leqslant n
$$

$\left(x_{0}<l\right.$ if $\left.i=0\right)$ and

$$
-i(a)=\left(\alpha_{0}, \ldots, \alpha_{i-1}, \alpha_{i}-1, \alpha_{i+1}, \ldots, \alpha_{n}\right), \quad 1 \leqslant i \leqslant n .
$$

For a function $v^{a}$ defined for $a \in A$ we define the difference operators

$$
\begin{gathered}
v^{a 0}=\left(v^{0(a)}-v^{a}\right) / k, \quad v^{a i}=\left(v^{i(a)}-v^{-i(a)}\right) / 2 h, \\
v^{a i j}=\frac{s(i, j)}{2 h^{2}}\left(v^{i(a)}+v^{-i(a)}+v^{j(a)}+v^{-j(a)}-2 v^{a}-v^{i(-s(i, j) j(a))}-v^{-i(s(i, j) j(a) \prime}\right)
\end{gathered}
$$

$(i, j=1, \ldots, n)$, where $s(i, i)=1$ and, for $i \neq j$,

Thus, in particular,

$$
s(i, j)=\left\{\begin{aligned}
1 & \text { if } \partial f / \partial r_{i j} \leqslant 0 \\
-1 & \text { if } \partial f / \partial r_{i j} \geqslant 0
\end{aligned}\right.
$$

$$
v^{a i i}=\frac{1}{h^{2}}\left(v^{i(a)}-2 v^{a}+v^{-i(a)}\right) .
$$

For a function $w$ defined on $S$ we denote $w^{a}:=w\left(x^{a}\right)$. One can show that if $w \in C^{2}(S)$ then $w^{a i j} \rightarrow w_{x_{j} x_{j}}^{a}$ as $h \rightarrow 0$. Finally, let

$$
v^{a l}=\left(v^{a 1}, \ldots, v^{a n}\right), \quad v^{a I J}=\left(v^{a i j}\right)_{i, j=1}^{n} .
$$


We consider the following explicit difference scheme corresponding to problem (1):

$$
\begin{array}{rlrl}
v^{u 0} & =f\left(x^{a}, r^{a}, v^{a t}, t^{a l t}\right) & & \text { for } a \in A^{\prime}, \\
t^{a}=g^{a} & \text { for } \alpha_{0}=0 .
\end{array}
$$

Our main result is the following

Thforem. Let Assumptions I, II and III be satisfied. Suppose

$$
h \leqslant h_{0}, \quad d \leqslant k / h^{2} \leqslant 1 / 2 n L_{2},
$$

where $d=2 / \lambda$ and $0<h_{0} \leqslant 2 \varkappa / L_{1}$ if $L_{1}>0$ whereas $h_{0}$ may be any positive number if $L_{1}=0$. Then

$$
\left|u^{a}-v^{u}\right| \leqslant z^{a} \quad \text { for } a \in A,
$$

where $u^{a}$ and $v^{a}$ are the values at the nodal points of the solutions of problems (1) and (7) respectively, and

$$
\begin{gathered}
z^{a}=M \omega^{a} \Phi^{u}, \quad \omega^{a}=\mu(k, h)\left[\exp \left(L_{0} \alpha_{0} k\right)-1\right] / L_{0}, \\
\mu(k, h)=k / 2+\left[n L_{1} h^{2} / 6+5 n^{2} L_{2} h / 3\right] \exp \left(K_{0} h^{2}\right), \quad K_{0}=N K /(N-K) . \\
\left.\Phi^{a}=\sum_{i=1}^{n} \varphi_{i}^{u}, \quad \varphi_{i}^{a}=\exp _{1} N e^{i x_{0}^{k}} x_{i}^{2} h^{2}+\gamma_{0} \alpha_{0}\right\}, \\
\gamma=\max i_{i}^{2} \lambda^{-1}(1-p)^{-2} N \exp \left[2 N e^{\lambda T}\left(1+h_{0}^{2}\right)+\lambda T\right], \\
2 L_{2}\left[\exp \left(h_{0}^{2} N e^{\lambda T}\right)-1\right] / p h_{0}^{2},(\lambda N / 2) e^{\lambda T\}} .
\end{gathered}
$$

3. In this section we preserve all the assumptions of the theorem and prove several lemmas.

Lemma 1. We have

$$
\left|u^{a}-v^{a}\right| \leqslant 2 M D^{\alpha_{0}} \sum_{v=1}^{n} \exp \left(K e^{\lambda \alpha_{0}^{k}} \alpha_{v}^{2} h^{2}\right) \quad \text { for } a \in A .
$$

where

$$
D=D_{0} \exp \left\{(K / 2) e^{\lambda T}\right\}, \quad D_{0}=1+2 n+h_{0} L_{1} / 2 L_{2}+h_{0}^{2}\left(L_{0}+1\right) / 2 n L_{2} .
$$

Proof. We first show by induction that

$$
\left|v^{a}\right| \leqslant M D_{0}^{\alpha_{0}} \sum_{v=1}^{n} \exp \left\{K\left(\left|\alpha_{v}\right|+\alpha_{0}\right)^{2} h^{2}\right\}, \quad a \in A .
$$

If $\alpha_{0}=0,(12)$ follows from the growth condition imposed on $g$. Suppose that (12) holds for $\alpha_{0}=p$. Hence we get

$$
\begin{aligned}
& \left|v^{i(a)}\right|,\left|t^{-i(a)}\right|,\left|v^{i(-s)(i, j) j(a))}\right|,\left|v^{\cdots i(s(i, j) j(a))}\right| \\
& \leqslant M D_{0}^{p} \sum_{\nu=1}^{n} \exp \left\{K\left(\left|\alpha_{v}\right|+1+p\right)^{2} h^{2}\right\} .
\end{aligned}
$$


Taking advantage of (7), (8), (3) and (13), one can easily check that

$$
\left|v^{0(a)}\right| \leqslant M D_{0}^{p+1} \sum_{v} \exp \left\{K\left(\left|\alpha_{v}\right|+1+p\right)^{2} h^{2}\right\},
$$

i.e., (12) with $\alpha_{0}=p+1$. Now, since

$$
\left(\left|\alpha_{v}\right|+\alpha_{0}\right)^{2} \leqslant e^{\lambda \alpha_{0} k} \alpha_{v}^{2}+\alpha_{0}^{2} e^{\lambda x_{0}^{k}} /\left(e^{i \alpha_{0} k}-1\right)
$$

and $e^{i x_{0} k}-1 \geqslant i \alpha_{0} k \geqslant \lambda \alpha_{0} d h^{2}$, we get from (12)

$$
\left|v^{a}\right| \leqslant M D^{\alpha_{0}} \sum_{v=1}^{n} \exp \left(K e^{\lambda 0_{0}^{k}} \alpha_{v}^{2} h^{2}\right)
$$

(14) and (2) imply (10).

Lemma 2. For $a \in A$ we have the estimates

$$
\begin{gathered}
\left|u_{x_{0}}^{a}-u^{a 0}\right| \leqslant(k / 2) H\left(x^{a} ; M, K\right), \\
\left|u_{x_{i}}^{a}-u^{a i}\right| \leqslant\left(h^{2} / 6\right) H\left(x^{a} ; M, N\right) \exp \left(K_{0} h^{2}\right), \\
\left|u_{x_{i} x_{j}}^{a}-u^{a i j}\right| \leqslant(5 h / 3) H\left(x^{a} ; M, N\right) \exp \left(\mathrm{K}_{0} h^{2}\right) .
\end{gathered}
$$

Proof. This is obtained by using Taylor's formula and (2).

We define

$$
\eta_{a}(k, h)=f\left(x^{a}, u^{a}, u^{a I}, u^{a I J}\right)-u^{a 0}, \quad a \in A^{\prime} .
$$

Lemma 3.

$$
\left|\eta_{a}(k, h)\right| \leqslant \mu(k, h) H\left(x^{a} ; M, N\right) .
$$

Proof. By (18), (1) and the mean value theorem,

$$
\begin{aligned}
\mid \eta_{a}(k, h) & =\left|u^{a 0}-f\left(x^{a}, u^{a}, u^{a l}, u^{a l J}\right)-\left[u_{x_{0}}^{a}-f\left(x^{a}, u^{a}, u_{x}^{a}, u_{x x}^{a}\right)\right]\right| \\
& \leqslant\left|u^{a 0}-u_{x_{0}}^{a}\right|+\sum_{i}\left|\frac{\partial f}{\partial q_{i}}\right|\left|u^{a i}-u_{x_{i}}^{a}\right|+\sum_{i, j}\left|\frac{\partial f}{\partial r_{i j}}\right|\left|u^{a i j}-u_{x_{i} x_{j}}^{a}\right|
\end{aligned}
$$

Applying Lemma 2 and (3), we get (19).

Lemma 4. The function $\Phi^{a}$ satisfies the inequality

$$
\Lambda\left(\Phi^{a}\right):=L_{2} \sum_{i, j=1}^{n}\left|\Phi^{a i j}\right|+L_{1} \sum_{i=1}^{n}\left|\Phi^{a i}\right|-\Phi^{a 0} \leqslant 0 \quad \text { for } a \in A^{\prime} .
$$

Proof. It is sufficient to prove that $\varphi_{i}^{a}$ satisfies the inequality

$$
L_{2}\left|\varphi_{i}^{a i i}\right|+L_{1}\left|\varphi_{i}^{a i}\right|-\varphi_{i}^{a 0} \leqslant 0 \quad(1 \leqslant i \leqslant n) .
$$

Setting $C=N e^{\lambda \alpha 0^{k}}$, we get

$$
\varphi_{i}^{a 0} / \varphi_{i}^{a}=\frac{1}{k}\left\{\exp \left[C\left(e^{\lambda k}-1\right) \alpha_{i}^{2} h^{2}+\gamma k\right]-1\right\} .
$$


Hence, by the inequalities $e^{s} \geqslant 1+s$ and $k \geqslant d h^{2}$ we obtain

$$
\varphi_{i}^{a 0} / \varphi_{i}^{a} \geqslant \gamma \exp \left(2 C \alpha_{i}^{2} h^{4}\right)+\lambda C \alpha_{i}^{2} h^{2} .
$$

Further, we have

$$
\left|\varphi_{i}^{a i}\right| / \varphi_{i}^{a}=h^{-1} e^{c h^{2}} \sinh \left(2 C\left|x_{i}\right| h^{2}\right) .
$$

Since $\sinh s \leqslant s e^{s}(s \geqslant 0)$, we obtain

$$
\begin{aligned}
L_{1}\left|\varphi_{i}^{a j}\right| / \varphi_{i}^{a} \leqslant & 2 L_{1} C\left|\alpha_{i}\right| h \exp \left[C\left(1+x_{i}^{2} h^{4}+h^{2}\right)\right] \\
\leqslant & L_{1}^{2} \lambda^{-1}(1-p)^{-1} C \exp \left[2 C\left(1+x_{i}^{2} h^{4}+h^{2}\right)\right]+ \\
& +\lambda(1-p) C \alpha_{i}^{2} h^{2} .
\end{aligned}
$$

Comparing (23) with (22), we find, by the definition of $\gamma$,

$$
L_{1}\left|\varphi_{i}^{a i}\right|-(1-p) \varphi_{i}^{u 0} \leqslant 0 .
$$

Let

$$
\psi\left(\alpha_{i}\right):=\left(L_{2}\left|\varphi_{i}^{a i i}\right|-p \varphi_{i}^{a 0}\right) / \varphi_{i}^{a}, \quad 1 \leqslant i \leqslant n .
$$

It is sufficient to show that $\psi\left(\alpha_{i}\right) \leqslant 0$ because this and (24) yield (21). We have

$$
\psi(\alpha)=\frac{2 L_{2}}{h^{2}}\left\{e^{C h^{2}} \cosh \left(2 C x h^{2}\right)-1\right\}-\frac{p}{k}\left\{\exp \left[C\left(e^{\lambda k}-1\right) \alpha^{2} h^{2}+\gamma k\right]-1\right\} .
$$

It is easy to check that $\psi(0) \leqslant 0$. Since $\psi(-\alpha)=\psi(\alpha)$, it suffices to show that $\psi^{\prime}(x) \leqslant 0$ for $x \geqslant 0$. Making use of the inequalities $e^{s} \geqslant 1+s, k \geqslant d h^{2}$, $\sinh s \leqslant s e^{s}(s \geqslant 0)$ and $s \leqslant s^{2} / 2 C+C / 2$, we derive

$$
\psi^{\prime}(\alpha)=4 L_{2} C s \exp \left(s^{2} / 2 C+C / 2+C h^{2}\right)-\lambda p s \exp \left(s^{2} / 2 C+\gamma d h^{2}\right)
$$

where $s=2 C \alpha h^{2}$. Further, the definitions of $\lambda$ and $\gamma$ imply $\psi^{\prime}(\alpha) \leqslant 0$, q.e.d.

LEMMA 5. Function $z^{a}$ satisfies the inequality

$$
\Lambda\left(z^{a}\right)+L_{0} z^{a}+\left|\eta_{a}(k, h)\right| \leqslant 0, \quad a \in A^{\prime} .
$$

Proof. It is easy to see that

$$
\begin{gathered}
z^{\alpha i}=M \omega^{a} \Phi^{a i}, \quad z^{a i j}=M \omega^{a} \Phi^{a i j}, \quad z^{a 0}=M \omega^{a 0} \Phi^{a}+M \omega^{0(a)} \Phi^{a 0}, \\
\omega^{0(a)} \geqslant \omega^{a}, \quad \Phi^{a 0} \geqslant 0, \quad \omega^{a 0} \geqslant L_{0} \omega^{a}+\mu(k, h) .
\end{gathered}
$$

These inequalities and Lemmas 3 and 4 imply Lemma 5.

4. Proof of the theorem. We set

$$
w^{a}=u^{a}-v^{a}, \quad F^{a}=\left|w^{a}\right|-z^{a}, \quad F_{1}^{a}=w^{a}-z^{a}, \quad F_{2}^{a}=-w^{a}-z^{a} .
$$

Let $A_{\varrho}=\left\{a \in A:\left|\alpha_{i}\right|<\varrho, i=1, \ldots, n_{\}}\right.$. By Lemma 1 there is an integer $\varrho$ such that $F^{a} \leqslant 0$ for $a \in A \backslash A_{\boldsymbol{e}}$. We shall show that $F^{a} \leqslant 0$ for $a \in A_{\boldsymbol{e}}$. Suppose 
the contrary. Then there would exist a multi-index $a \in A_{e}$, with $\alpha_{0}>0$, such that $F^{a}>0$ and $F^{b} \leqslant 0$ for all $b=\left(\beta_{0}, \beta_{1}, \ldots, \beta_{n}\right) \in A_{e}$ with $\beta_{0}<\alpha_{0}$. Take $b$ so that $o(b)=a$. It is sufficient to show that $F_{i}^{a} \leqslant 0$ and $F_{2}^{a} \leqslant 0$. We have

$$
F_{1}^{a}=F_{1}^{b}+k F_{1}^{b 0}=F_{1}^{b}+k\left(u^{b 0}-v^{b 0}-z^{b 0}\right) \text {. }
$$

Hence, by (18), (7) and the mean value theorem,

$$
\begin{aligned}
& F_{1}^{a} \leqslant F_{1}^{b}+k\left\{\left|\eta_{b}\right|+\left|\frac{\partial f}{\partial u}\right|\left|w^{b}\right|\right. \\
&=F_{1}^{b}+k\left\{\frac{\partial f}{\partial} \frac{\partial f}{\partial q_{i}} w^{b i}+F_{i, j} \frac{\partial f}{\partial r_{i j}} w^{b i j}-\sum_{i} \frac{\partial f}{\partial q_{i}} F_{1}^{b i}+\sum_{i, j} \frac{\partial f}{\partial r_{i j}} F_{1}^{b i j}\right\}+ \\
&+k\left\{\left|\eta_{b}\right|+\left|\frac{\partial f}{\partial u}\right| z^{b}+\sum_{i} \frac{\partial f}{\partial q_{i}} z^{b i}+\sum_{i, j} \frac{\partial f}{\partial r_{i j}} z^{b i j}-z^{b 0}\right\} .
\end{aligned}
$$

The expression in the last bracket is non-positive. Also $|\partial f / \partial u| F^{b} \leqslant 0$. Consequently,

$$
\begin{aligned}
& F_{1}^{a} \leqslant F_{1}^{b}+k\left\{\frac{1}{2 h} \sum_{i} \frac{\partial f}{\partial q_{i}}\left(F_{1}^{i(b)}-F_{1}^{-i(b)}\right)+\frac{1}{h^{2}} \sum_{i} \frac{\partial f}{\partial r_{i i}}\left(F_{1}^{i(b)}-2 F_{1}^{b}+F_{1}^{-i(b)}\right)+\right. \\
&+\frac{1}{2 h^{2}} \sum_{i, j(i \neq j)}\left|\frac{\partial f}{\partial r_{i j}}\right|\left(-F_{1}^{i(b)}-F_{1}^{-i(b)}-F_{1}^{j(b)}-F_{1}^{-j(b)}+\right. \\
&\left.\left.+2 F_{1}^{b}+F_{1}^{i(-s(i, j) j(b))}+F_{1}^{-i(s(i, j) j(b))}\right)\right\} .
\end{aligned}
$$

Hence, rearranging the terms, we find (cf. [9])

$$
\begin{aligned}
F_{1}^{a} \leqslant\left(1-\frac{2 k}{h^{2}} \sum_{i=1}^{n} \frac{\partial f}{\partial r_{i i}}\right) & F_{1}^{b}+\frac{k}{h} \sum_{i=1}^{n}\left[\frac{1}{h}\left(\frac{\partial f}{\partial r_{i i}}-\sum_{j=1, j \neq i}^{n}\left|\frac{\partial f}{\partial r_{i j}}\right|\right)+\frac{1}{2} \frac{\partial f}{\partial q_{i}}\right] F_{1}^{i(b)}+ \\
& +\frac{k}{h} \sum_{i=1}^{n}\left[\frac{1}{h}\left(\frac{\partial f}{\partial r_{i i}}-\sum_{j=1, j \neq i}^{n}\left|\frac{\partial f}{\partial r_{i j}}\right|\right)-\frac{1}{2} \frac{\partial f}{\partial q_{i}}\right] F_{1}^{-i(b)}+ \\
& +\frac{k}{2 h^{2}} \sum_{i, j(i \neq j)}\left|\frac{\partial f}{\partial r_{i j}}\right|\left(2 F_{1}^{b}+F_{1}^{i(-s(i, j) j(b))}+F_{1}^{-i(s(i, j) j(b))}\right) .
\end{aligned}
$$

The values $F_{1}^{b}, F_{1}^{i(b)}, F_{1}^{-i(b)}, F_{1}^{i(-s(i, j) j(b))}, F_{1}^{-i(s(i, j) j(b))}$ are non-positive. Making use of (3), (4) and (8), we obtain $F_{1}^{a} \leqslant 0$. The inequality $F_{2}^{a} \leqslant 0$ can be shown similarly.

Note finally that if $L_{1}=0$ one can take $p=1$ and repeat the proof, which now becomes simpler and the error less. Thus the proof is completed.

Remark 1. Our theorem related to linear equations requires the assumption that the coefficients at the second order mixed derivatives have a constant sign. However, it follows from the proof that in the linear case this assumption is superfluous.

Remark 2. The above result can easily be extended to the Cauchy 
problem for a weakly coupled system of the form

$$
\begin{array}{ll}
u_{v, x_{0}}=f_{v}\left(x, u, u_{v, x}, u_{v, x x}\right) & \text { in } S, \\
u_{v}(x)=g_{v}(x) & \text { for } x_{0}=0, \quad v=1, \ldots, m,
\end{array}
$$

where $u=\left(u_{1}, \ldots, u_{m}\right), u_{v, x}=\left(u_{v, x_{1}}, \ldots, u_{v, x_{n}}\right), u_{v, x x}=\left(u_{v, x_{i} x_{j}}\right)_{i, j=1}^{n}$.

Suppose that every function $f_{v}$ satisfies Assumption II except that the first inequality in (3) is replaced by $\left|\partial f_{v} / \partial u_{s}\right| \leqslant L(v, s=1, \ldots, m)$. Then the above theorem remains valid for each component $u_{v}$ if we replace $L_{0}$ by $m L$. The proof merely undergoes slight obvious changes.

\section{References}

[1] D. G. Aronson, On the stability of certain finite difference approximations to parabolic systems of differential equations, Num. Math. 5 (1963), 118-137.

[2] L. Collatz, The numerical treatment of differential equations, English translation: P. G. Williams, Springer, Berlin-Heidelberg-New York 1966.

[3] A. Fitzke, Method of difference inequalities for parabolic equations with mixed derivatives, Ann. Polon. Math. 31 (1975), 121-129.

[4] F. John, On integration of parabolic equations by difference methods, Comm. Pure Appl. Math. 5 (1952), 155-211.

[5] L. I. K a my nin, On the applicability of a finite difference method to the solution of the heat equation, II, Russian lzv. Acad. Nauk SSSR Ser. Mat. 17 (1953), 249-268.

[6] Z. Kowalski, On the difference method for a nonlinear system of parabolic differential equations without mixed derivatives, Bull. Acad. Polon. Sci., Sér. Sci. Math. Astr. Phys. 16 (1968), 303-310.

[7] - A difference method for a non-linear system of elliptic equations with mixed derivatives, Ann. Polon. Math. 38 (1980), 229-243.

[8] S. Lojasiewicz, Sur le problème de Cauchy pour les systèmes d'équations aux dérivées partielles du premier ordre dans le cas hyperbolique de deux variables indépendantes, ibidem 3 (1956), 87-117.

[9] M. Malec, Sur une certaine inégalité aux différences finies du second ordre, Bull. Acad. Polon. Sci., Sér. Sci. Math. Astr. Phys. 22 (1974), 503-506.

[10] -, Methode de différences finies pour une équation différentielle partielle avec dérivées mixtes, ibidem 22 (1974), 561-576.

[11] A. Pliś, On difference inequalities corresponding to partial differential inequalities of the first order, Ann. Polon. Math. 20 (1968), 179.181.

[12] A. Voigt, Line method approximations to the Cauchy prohleon for inonlinear purabolic differential equations, Num. Math. 23 (1974), 2336.

[13] W. Walter, Approximation für das Cauchy problem bei parabolischen Differentialgleichungen mit Linienmethode, ISNM vol. 10, Abstract spaces ${ }^{\bullet}$ and approximation, 139-145, Basel: Birkhauser 1969.

[14] -, Differential and integral inequalities, Springer, Berlin- Heidelberg New York 1970. 